\title{
XXXIV. Observations on the singular figure of the planet Saturn
}

\section{William Herschell LL.D. F.R.S.}

To cite this article: William Herschell LL.D. F.R.S. (1805) XXXIV. Observations on the singular figure of the planet Saturn , Philosophical Magazine Series 1, 23:90, 147-153, DOI: $10.1080 / 14786440508562505$

To link to this article: http://dx.doi.org/10.1080/14786440508562505

曲 Published online: 18 May 2009.

Submit your article to this journal $[\pi$

Џ Article views: 2

Q View related articles $\asymp$ 
100 grains of the stone to be examined in very fine powder, must be fused for about half an hour, at a strong red heat, in a crucible of platina or silver, with 200 grains of boracic acid.

An ounce and half of nitric acid, diluted with seven or eight times its quantity of water, must be digested upon the fused mass till the whole is decomposed.

The fluid must be evaporated till its quantity is reduced to an ounce and half or two ounces.

If the stone contain silex, this earth will be separated in the process of solution and evaporation; and it must be collected upon a filter, and washed with distilled water till the boracic acid and all the saline matter is separated from it.

The fluid, mixed with the water that has passed through the filter, must be evaporated, till it is reduced to a convenient quantity, such as that of half a pint ; when it must be saturated with carbonate of ammonia, and boiled with an excess of this salt, till all the materials that it contains, capable of being precipitated, have fallen to the bottom of the vessel.

The solution must then be separated by the filter, and the earths and metallic oxides retained.

It must be mixed with nitric acid till it tastes strongly sour, and evaporated till the boracic acid appears free.

The fluid must be passed through the filter, and subjected to evaporation till it becomes dry ; when, by exposure to a heat equal to $450^{\circ}$ Fahrenheit, the nitrate of ammonia will be decomposed, and the nitrate of potash or soda will remain in the vessel.

It will be unnecessary for me to describe minutely the method of obtaining the remaining earths and metallic oxides free from each orher, as I have used the common processes. I have separated the alumine by solution of potash, the lime by sulphuric acid, the oxide of iron by succinate of ammonia, the manganese by hydrosulphuret of potash, and the magnesia by pure soda.

XXXIV. Olservations on the singular Figure of the Planet Salurn. By William Herschel., LL.D. F.R.S.*

T

HERE is not perhaps another object in the heavens that presents us with such a variety of extraordinary phænomena

* From the Transactions of the Royal Suciety for 1805.

$\mathrm{K} 2$ 
as the planet Saturn : a magnificent globe, encompassed by a stupendous double ring; attended by seven satellites; ornamented with equatorial belts; compressed at the poles; turning upon its axis ; mutually eclipsing its ring and satellites, and eclipsed by them ; the most distant of the rings also turning upon its axis, and the same taking place with the furthest of the satellites; all the parts of the system of Saturn occasionally reflecting light to each other; the rings and moons illuminating the nights of the Saturnian; the globe and satellites enlightening the dark parts of the rings; and the planet and rings throwing back the sun's beams npon the moons, when they are deprived of them at the time of their conjunctions.

It must be confessed that a detail of circumstances like these appears to leave hardly any room for addition, and yet the following observations will prove that there is a singularity left, which distinguishes the figure of Saturn from that of all the other planets.

It has already been mentioned on a former occasion, that so far back as the year $17 \% 6$ I perceived that the body of Saturn was not exactly round; and when I found in the year 1781 that it was fiattened at the poles at least as much as Jupiter, I was insensibly diverted from a more critical attention to the rest of the figure. Prepossessed with its being spheroidical, I measured the equatorial and polar diameters in the year 1789 , and supposed there could be no other particularity to remark in the figure of the planet. When I perceived a certain irregularity in other parts of the body, it was generally ascribed to the interference of the ring, which prevents a complete view of its whole contour; and in this error I might still have remained, had not a late examination of the powers of my 10-feet telescope convipced me that I ought to rely with the greatest confidence upon the tiuth of its representations of the most minute objects I inspected.

The following observations, in which the singular figure of Saturn is fully investigated, contain many remarks on the rest of the appearances that may be seen when this beautiful planet is examined with attention; and though thcy are not immediately necessary to my present subject, I thought it right to retain them, as they show the degree of distinctness and precision of the action of the telescope, and the clearness of the atmosphere at the time of observation.

April 12, 1805. With a new 7 -feet mirror of extraor- 
dinary distinctness, I examined the planet Saturn. The ring reflects more light than the body, and with a power of 570 the colour of the body becomes yellowish, while that of the ring remains more white. This gives us an opportunity to distinguish the ring from the body, in that part where it crosses the disk, by means of the difference in the colour of the reflected light. I sav the quintuple belt, and the flattening of the body at the polar regions; I could also perceive the vacant space between the two rings.

The flattening of the polar regions is not in that gradual inanner as with Jupiter, it seems not to begin till at a high latitude, and there to be more sudden than it is towards the poles of Jupiter. I have often made the same observation before, but do not remember to have recorded it any where.

April 18; 10-fect reflector, power 300 . The air is very favourable, and I see the planet extremely well defined. The shadow of the ring is very black in its extent over the disk south of the ring, where $I$ see it all the way with great distinctness.

The usual belts are on the body of Saturn; they cover a much larger zone than the belts on Jupiter generally take up, as may be seen in the figure I have given in Plate IX.; and also in a former representation of the same belts in $1794^{*}$.

The figure of the body of Saturn, as I see it at present, is certainly different from the spheroidical figure of Jupiter. The curvature is greatest in a high latitude.

I took a measure of the situation of the four points of the greatest curvature, with my angular micrometer, and power 527. When the cross of the micrometer passed through all the four points, the angle which gives the double latitude of two of the points, one being north, the other south of the ring, or equator, was $93^{\circ} 16^{\prime} \ldots$ The latitude therefore of the four points is $46^{\circ} 38^{\prime}$; it is there the greatest curvature takes place. As neither of the cross wires can be in the parallel, it makes the measure so difficult to take, that very great accuracy cannot be expected.

The most northern belt comes up to the place where the ring of Saturn passes behind the body, but the belt is bent in a contrary direction, being concave to the north, on account of its crossing the body on the side turned towards us, and the north pole being in view.

There is a very dark, but narrow shadow of the body

* See Phil. Trans. for 1794, Table VI. page 52.

$\mathrm{K} 3$ upon 


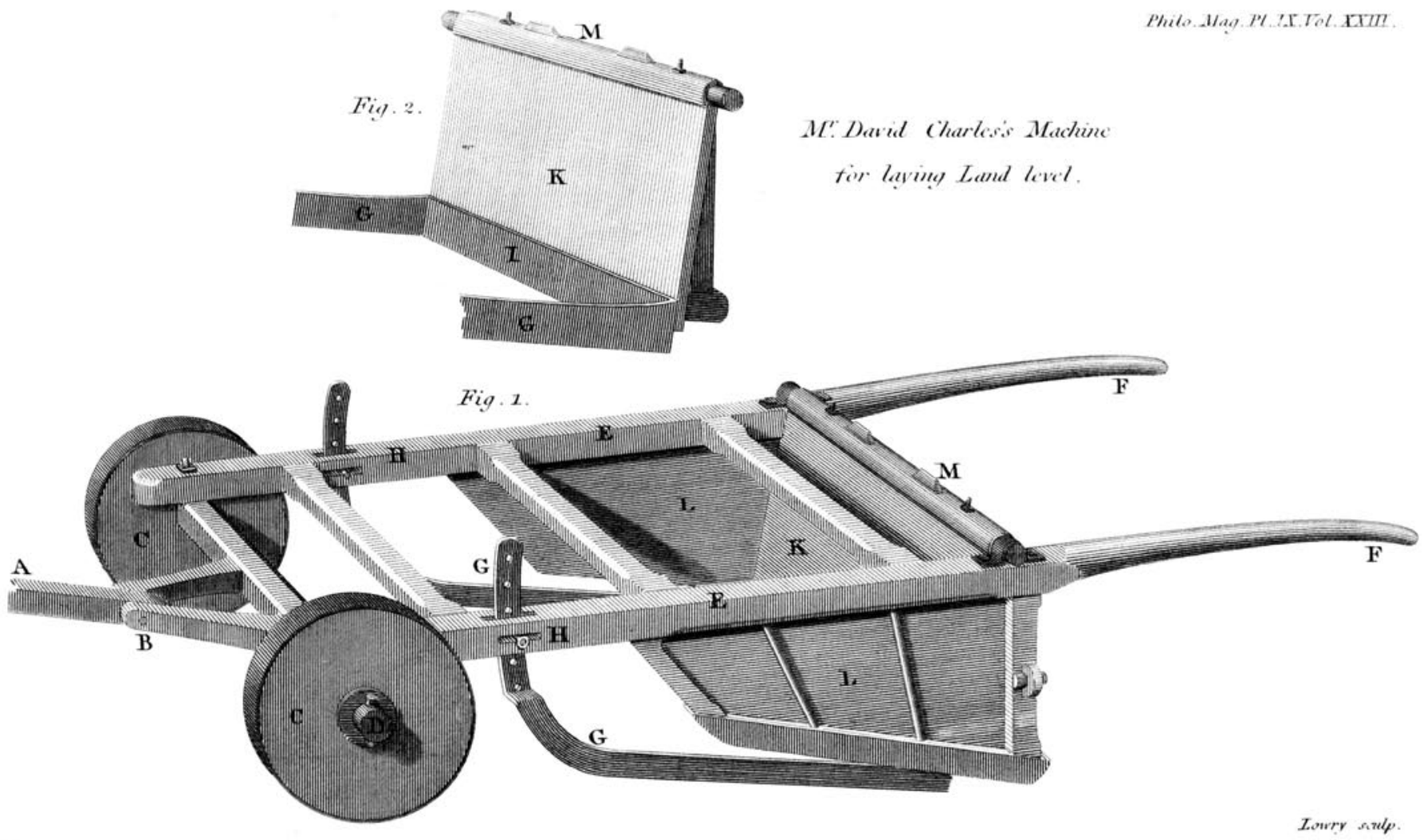


upon the following part of the ring, which as it were cuts off the ring from the body.

The shadow of the ring on the body, which I see south of the ring, grows a little broader on both sides near the maryin of the disk.

The division between the two rings is dark, like the vacant space between the ansæ, but not black like the shadow I have described.

There are four satellites on the preceding side near the ring; the largest and another are north-preceding; the other two are nearly preceding.

April 19. I viewed the planet Saturn with a new 7 -feet telescope, both mirrors of which are very perfect. I saw all the phænomena as described last night, except the satellites, which had changed their situation; four of them being on the following side. This telescope however is not equal to the 10-feet one.

The remarkable figure of Saturn admits of no doubt : when our oarticular attention is once drawn to an object, we see things at first sight that would otherwise have escaped our notice.

10-feet reflector, power 400. The night is beautifully, clear, and the plant near the meridian. The figure of Saturn is somewhat like a square or rather parallelogram, with the four corners rounded off deeply, but not so much as to bring it to a spheroid. I see it in perfection.

The four satellites that were last night on the preceding, are now on the following side, and are very bright.

I took a measure of the position of the four points of the greatest curvature, and found it $91^{\circ} 29^{\prime}$. This gives their latitude $45^{\circ} 44^{\prime}, 5$. I believe this measure to be pretty accurate. I set first the fixed thread to one of the lines, by keeping the north-preceding and south-following two points in the thread; then adjusted the other thread in the same manner to the south-preceding and north-following points.

May 5, 1805. I directed my 20-feet telescope to Saturn, and, with a power of about 300 , saw the planet perfectly well defined, the evening being remarkably clear. The shadow of the ring on the body is quite black. All the other phænomena are very distinct.

The figure of the planet is certainly not spheroidical, like that of Mars and Jupiter. The curvature is less on the equator and on the poles than at the latitude of about 45 degrees. The equatorial diameter is however considerably greater than the palar. 
In order to have the testimony of all my instruments, on the subject of the structure of the planet Saturn, I had prepared the 40-feet reflector for observing it in the meridian. 1 used a magnifying power of 360 , and saw its form exactly as $I$ had seen it in the 10- and go-feet instruments. The planet is flattened at the poles, but the spheroid that would arise from this flattening is morlified by some other cause, which I suppose to be the attraction of the ring. It resembles a parallelogram, one side whereof is the equatorial, the other the polar diameter, with the four corners rounded off so as to leave both the equatorial and polar regions flatter than they would be in a regular spheroidical figure.

The planet Jupiter being by this time got up to a considerable altitude, I viewed it alternately with Saturn in the 10-feet reflector, with a power of 500 . The oullines of the figure of Saturn are as described in the observation of the 40-feet telescope; but those of Jupiter are such as to give a greater curvature both to the polar and equatorial regions than takes place at the poles or equator of Saturn which are comparatively much flatter.

May 12. I viewed Saturn and Jupiter alternately with my large 10-feet telescope of 24 inches aperture : and saw plainly that the former planet differs much in figure from the latter.

The temperature of the air is so changeable that no large mirror can act well.

May 13. 10-feet reflector, power 300. The shadows of the ring upon the body, and of the body upon the ring, are very black, and not of the dusky colour of the heavens about the planet, or of the space between the ring and planet, and between the two rings. The north-following part of the ring, close to the planet, is as it were cut off by the shadow of the body: and the shadow of the ring lies south of it, but close to the projection of the ring.

The planet is of the form described in the observation of the 40 -feet telescope; I see it so distinctly that there can be no doubt of it. By the appearance I should think the points of the greatest curvature not to be so far north as $\mathbf{4 5}$ degrees.

The evening being very calm and clear, I took a measure of their situation, which gives the latitude of the greatest curvature $45^{\circ} 2 \mathrm{~J}^{\prime}$. A second measure gives $45^{\circ} 4 \mathrm{I}^{\prime}$.

Jupiter being now at a considerable altitude, I have viewed it alternately with Saturn. The figure of the two planets is decidedly different. The flattening at the poles and on the K 4 equator 


\section{Olservations on the Figure of the Planet Saturn.}

equator of Saturn is much greater than it is on Jupiter, but the curvature at the latitude of from 40 to $48^{\circ}$ on Jupiter is less than on Saturn.

I repeated these alternate observations many times, and the oftener I compared the two planets together, the more striking was their different structure.

May 26. 10-feet reflector. With a parahel thread micrometer and a magnifying power of 400 , I took two measures of the diameter of the points of greatest curvature. A mean of them gave 64,3 divisions $=11^{\prime \prime}, 98$. After this, I took also two measures of the equatorial diameter, and a mean of them gave 60,5 divisions $=11^{\prime \prime}, 27$; but the equatorial measures are probably too small.

To judge by a view of the planet, I should suppose the latitude of the greatest curvature to be less than 45 degrees. The eye will also distinguish the difference in the three diameters of Saturn. That which passes through the points of the grcatest curvature is the largest; the equatorial the next, and the polar diameter is the smallest.

May 27. The evening being very favourable, I took again two measures of the diameter between the points of greatest curvature, a mean of which was 63,8 divisions $=11^{\prime \prime}, 88$. Two measures of the equatorial diameter gave 61,3 divisions $=11^{\prime \prime}, 44$.

June 1. It occurred to me that a more accurate measure might be had of the latitude in which the greatest curvature takes place, by setting the fixed thread of the micrometer to the direction of the ring of Saturn, which may be done with great accuracy. The two following measures were taken in this manner, and are more satisfactory than I had taken before. The first gave the latitude of the south-preceding point of greatest curvature $43^{\circ} 26^{\prime}$; and the second $43^{\circ} 13^{\prime}$. A mean of the two will be $43^{\circ} 20^{\prime}$.

June 2. I viewed Jupiter and Saturn alternately with a magnifying power of only 300 , that the convexity of the eye-glass might occasion no deception, and found the form of the two planets to differ in the manner that has been described.

With 200 I saw the difference very plainly; and even with 160 it was sufficiently visible to admit of no doubt. These low powers show the figure of the planets perfectly well, for as the field of view is enlarged, and the motion of the objects in passing it lessened, we are more at liberty to fix our attention upon them.

I compared the telescopic appearance of Saturn with a figure drawn by the measures I have taken, combined with 
the proportion between the equatorial and polar diameters determined in the year 1789 * ; and found that, in order to be a perfect resemblance, my figure required some small reduction of the longest diameter, so as to bring it nearly to agree with the measures taken the 27th of May. When I had made the necessary alteration, my artificial Saturn was again compared with the telescopic representation of the planet, and I was then satisfied that it had all the correctness of which a judgment of the eye is capable. An exact copy of it is given in Plate VII. The dimensions of it in proportional parts are,

$\begin{array}{llr}\text { The diameter of the greatest curvature } & - & 36 \\ \text { The equatorial diameter } & - & 35 \\ \text { The polar diameler } & - & 32 \\ \text { Latitude of the longest diameter } & - & \mathbf{4 3}^{\circ} \mathbf{2 0 ^ { \prime }}\end{array}$

The foregoing observations of the figure of the body of Saturn, will lead to some intricate researches, by which the quantity of matter in the ring, and its solidity, may be in some measure ascertained. They also afford a new instance of the effect of gravitation on the figure of planets; for in the case of Saturn, we shall have to consider the opposite influence of two centripetal and two centrifugal forces: the rotation of both the ring and planet having been ascertained in some of my former papers.

XXXV. Experiments and Observations upon the Contraction of Water by Heat at low Temperatures. By Tromas Charles Hope, M.D. F.R.S. Edin., Professor of Chemistry in the University of Edinlurgh*.

To the general law, that bodies are expanded by, heat, and contracted by cold, water at the point of congelation, and for some degrees of temperature above it, seems to afford a very singular and curious exception.

The circumstances of this remarkable anomaly have been for some time believed to be the following:

When heat is applied to water ice-cold, or at a temperature not far distant, it causes a diminution in the bulk of the fluid. The water contracts, and continues to contract, with the augmentation of temperature, till it reaches the

* See Phil. Trans for 179́o, p. 17.

+ From the Transactions of the Royal Society of Edinlurgh, part ii. of vol. v. 


$$
\infty
$$

